\title{
Pharmacokinetics of Digoxin: Interpreting Bioavailability
}

\author{
N. SANCHEZ, L. B. SHEINER, H. HALKIN, K. L. MELMON
}

Since each of these accepted methods has its weaknesses if used singly, the bioavailability of digoxin or other drugs is better assessed if the three methods are compared in the same subjects.

\section{Summary}

Three subjects were first given a digoxin tablet in the fasting state and subsequently received the same formulation in the fed state, to simulate a spurious oral bioavailability difference. As expected, when measured by peak serum digoxin concentration as well as by area under the serum digoxin concentration-time curve the bioavailability of digoxin appeared to be higher in the fasting state than in the fed state. However, when measured by cumulative five-day urinary excretion of digoxin bioavailability was identical in both conditions.

This simple experiment illustrates the fact that the estimation of bioavailability is subject to considerable methodological error, of which the clinician must be aware when evaluating reports on drug bioavailability in general and of digoxin in particular.

\section{Introduction}

The concept of drug bioavailability is important because the effect of a preparation given to a patient correlates more closely with the plasma concentration of drug than with the dose taken -that is, the drug must reach its site of action in order to exert its effect. A common definition of bioavailability is the rate at which active drug is absorbed into the systemic circulation from its formulary form and the extent of its absorption.

This study will emphasize that the two variables of bioavailability, rate and extent of absorption, are independent of each other. The former determines the speed with which peak drug plasma concentration is achieved, but is not related to completeness of absorption. The latter relates to the total amount of drug absorbed into the blood stream.

A number of recent studies have investigated the bioavailability of various types and brands of oral digoxin (Lindenbaum et al., 1971; Manninen et al., 1971; Binnion and McDermott, 1972; Hibble et al., 1972; Wagner et al., 1973). Bioavailability may be misjudged if inaccurate conclusions are drawn from comparative peak serum concentrations of drug. Plotting serum drug concentrations versus time affords only one means of quantitating bioavailability. The time needed to achieve peak concentration indicates the rate of absorption of drug. A second estimate of bioavailability is given by the total area under the serum drug concentration-time curve (or the integral of the function describing the serum drug concentration) which indicates the total extent of drug absorbed into the body (Dittert et al., 1972; Wagner et al., 1973). A third estimate is given by measuring the cumulative total excretion of the free drug and its metabolites (Dittert et al., 1972; Huffman and Azarnoff, 1972).

\section{Department of Medicine, Division of Clinical Pharmacology, University of California Medical Center, San Francisco, California 94143}

N. SANCHEZ, M.D., Post-Doctural Research Fellow

L. B. SHEINER, M.D., Assistant Professor of Medicine and Clinical Pathology

H. HALKININ, M.D., Merck International Fellow in Clinicai Pharmacology K. L. MELMON, M.D., Professor of Medicine and Pharmacology This study gives an example of the possible practical problems arising from incomplete evaluation of bioavailability of digoxin versus the clinical usefulness of measuring it in three ways.

The experimental setting was designed to produce spurious apparent differences in bioavailability from the same oral formulation when the estimation of bioavailability was based on serum data alone.

\section{Materials and Methods}

Six healthy, ambulatory male volunteers, aged 29-33 years and not taking medication, each ingested one $0.25 \mathrm{mg}$ digoxin tablet (Burroughs-Wellcome, Lanoxin $0.25 \mathrm{mg}$, Lot $770 \mathrm{~B}$ ) at 08.00 hours after a 12 - to 16-hour overnight fast. The subjects were allowed to resume normal eating and working three hours after tablet ingestion. Consecutive 24-hour urine collections were obtained by each subject for five days after the drug was taken. In three subjects blood samples were drawn at $0.5,1.0,1.5,2.0$, and 3.0 hours after ingestion. (Sampling was not continued beyond three hours in order to ensure that it had been limited to the distribution phase.) After a nine-day interval the experiment was repeated, the digoxin tablet being ingested by each subject immediately after a usual breakfast. Completeness of the urine collections was ascertained by determining the daily urinary excretion of creatinine by each subject.

Laboratory Procedures.-The digoxin in urine, serum, and tablets was measured by radioimmunoassay (Smith et al., 1969) utilizing a $20 \mu \mathrm{g} / \mathrm{ml}$ standard digoxin solution. Urinary creatinine was determined colorimetrically by a micromodification of the picric acid method (Oser, 1965).

Data Analysis.-Cumulative five-day urinary excretion of digoxin was calculated for each of the six subjects (five-day urine pool digoxin concentration $\times$ total five-day urine volume). The mean cumulative excretion of digoxin was calculated for all subjects and expressed as the fraction of the dose administered $(0.25 \mathrm{mg})$ that was excreted in the urine. The fractions excreted in the urine during both the fasting and fed-state experiments were compared by the paired $t$ test.

Serum digoxin concentration curves versus time were plotted by calculating the mean concentration of digoxin in serum at each time point in both experiments for the three subjects from whom blood samples were drawn. The areas under the two mean curves were calculated by the trapezoidal rule (Dittert et al., 1972) and compared by the $t$ test, as were the peak concentrations of digoxin in serum in each curve.

\section{Results}

Five randomly selected digoxin tablets were assayed from the lot used. Mean tablet digoxin content was $0.237 \mathrm{mg}$ with a standard deviation of $0.029 \mathrm{mg}$, representing $11.5 \%$ of the stated tablet content $(0.250 \mathrm{mg})$.

Average concentrations of digoxin in serum, average cumulative five-day urinary excretion of digoxin, average peak concentrations of digoxin in serum, and the average areas under the curves, for both fasting and postprandial digoxin ingestion are given in the table. Mean concentrations of digoxin in serum versus time are shown in fig. 1 . The comparisons for fasting and 
postprandial states of the mean areas under the concentration curves, the peak mean concentration of digoxin in serum, and mean cumulative urinary excretion are shown in fig. 2 .

Results of Tests expressed in $\mu \mathrm{g} / \mathrm{ml}$

\begin{tabular}{c|c|c}
\hline $\begin{array}{c}\text { Time of Sample } \\
\text { after Ingestion }\end{array}$ & $\begin{array}{c}\text { Fasting } \\
\text { (Mean } \pm \text { S.E. of Mean) }\end{array}$ & $\begin{array}{c}\text { Postprandial } \\
\text { (Mean } \pm \text { S.E. of Mean) }\end{array}$ \\
\hline 0.5 & $1.08 \pm 0.02^{*}$ & $0.19 \pm 0.05$ \\
1.0 & $0.88 \pm 0.16$ & $0.46 \pm 0.15$ \\
1.5 & $0.63 \pm 0.19$ & $0.48 \pm 0.20^{*}$ \\
2.0 & $0.42 \pm 0.02$ & $0.27 \pm 0.11$ \\
3.0 & $0.33 \pm 0.03$ & $0.15 \pm 0.02$ \\
\hline $\begin{array}{c}\text { Mean area under serum } \\
\text { concentration versus } \\
\text { time curve (ng hr/ml) }\end{array}$ & $1.78 \pm 0.10$ & \\
$\begin{array}{c}\text { Mean cumulative 5-day } \\
\text { excretion of digoxint }\end{array}$ & $0.39 \pm 0.07$ & $0.82 \pm 0.13$ \\
\end{tabular}

- Peak mean concentration of digoxin in serum

† Expressed as fraction of $0.250 \mathrm{mg}$ administered dose.

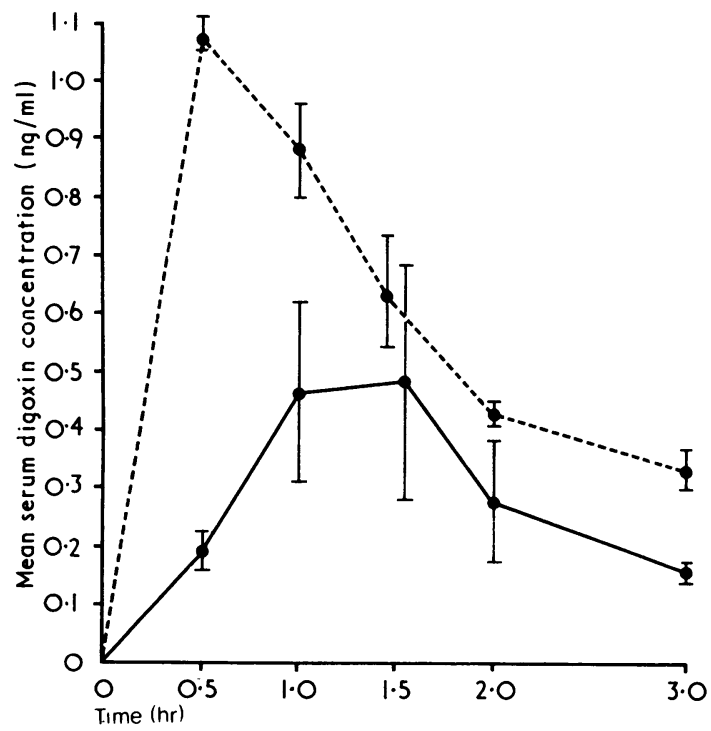

FIG. 1-Mean ( \pm S.E. of mean) serum digoxin concentrationtime curves in the fasting (broken line) and postprandial (solid line) states.

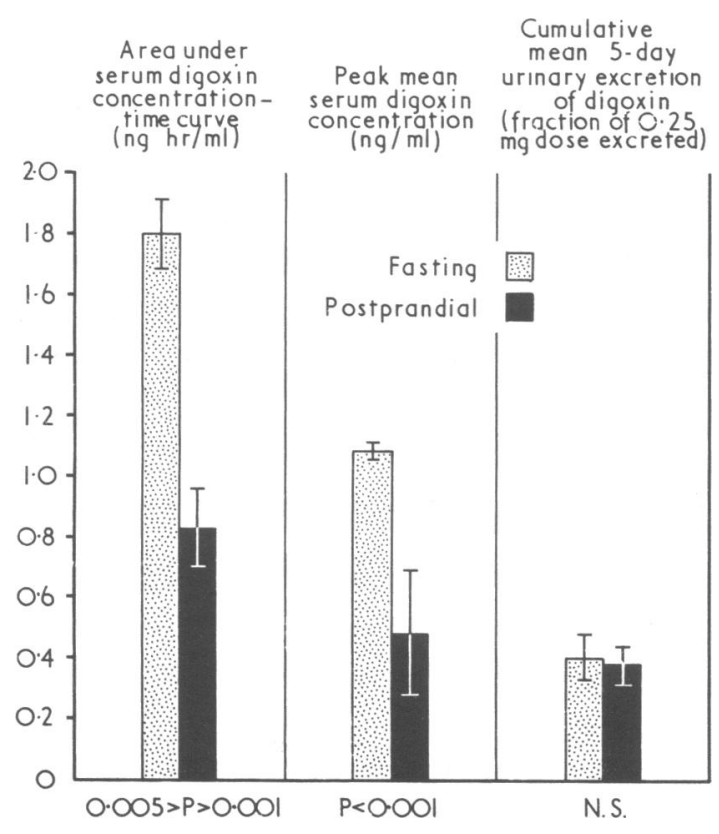

FIG. 2-Comparison of bioavailability as estimated by area under serum concentration-time curve, peak mean serum concentration, and cumulative urinary excretion of digoxin. N.S. = Not significant.

\section{Discussion}

As expected (White et al., 1971), digoxin bioavailability as reflected by peak serum concentration-that is, rate of absorption-was significantly greater when the subjects took the drug while fasting than when they took it postprandially. In addition, the extent of absorption (represented by the area under the serum concentration-time curve) was seemingly greater when the drug was taken while fasting; yet no difference in the extent of bioavailability of digoxin between the fasting and fed states was found by the method of measuring cumulative urinary excretion.

After the oral administration of digitalis the serum concentration of the drug rises followed by a decrease in blood levels reflecting the continuing, but now predominant, distribution of the drug from the blood into tissues (including the myocardium). This phase of tissue equilibration is of varying duration lasting two to four hours after an intravenous dose and even longer after oral administration (Butler, 1972). In the case of intravenously administered digoxin the maximum pharmacological effect of the drug occurs at least one hour after serum levels have reached their peak (Deutscher et al., 1972). It is thus quite likely that the maximum pharmacological effect after oral administration does not correlate with peak serum digoxin concentrations. Thus, the peak serum concentration after absorption of digoxin is of secondary importance, both in determining its bioavailability and the optimal time of pharmacological effect. The extent of absorption is the factor of overriding clinical importance. It is the balance between this factor and elimination which determines the mean steady-state plasma and tissue concentrations of drug, which in turn determine the long-term pharmacological effect of the drug.

In both of our experimental settings (the fasting and postprandial) an apparent discrepancy was shown between extent of absorption as measured by area under the "blood concentration-time" curve and by cumulative urinary excretion. This discrepancy is superficial and is accounted for by the early termination of blood sampling at three hours, a common inappropriate practice in interpretation of blood concentrationtime curves. The extent of absorption is truly represented by the total area under the blood concentration-time curve only when the sampling period is extended well into the equilibration phase (Dittert et al., 1972). The area calculated from a curve that has been terminated prematurely can produce a falsely low estimate of the extent of absorption, caused by the amputation of the area under the late part of the curve, though it may be correlated with the area under the entire curve (Wagner et al., 1973).

Given a known rate and normally minor role of metabolic breakdown of digoxin (Doherty et al., 1971) plus the fact that in normal subjects elimination of digoxin is chiefly by renal excretion of unchanged drug (Doherty et al., 1971; Doherty et al., 1972), the amount of free drug excreted in the urine will be a good measure of the extent of its absorption from an oral formulation (Huffman and Azarnoff, 1972) if the same stipulation is followed as with measuring blood concentration-time curves. The period of sampling must be long enough, in this case, to cover the time required for excretion of almost all the drug that can be excreted. It has been shown that in normal subjects the urinary excretion of digoxin after a single oral dose is virtually complete in five days and consists of $40 \%$ of the administered dose (Doherty, 1968). Our results on the urinary excretion of digoxin are in excellent agreement with these findings $(39 \%$ of the dose being excreted in five days in both experiments).

In summary, we have seen that after oral administration of digoxin, the peak serum concentration of drug is relatively unimportant in the evaluation of bioavailability, and that statements on bioavailability related to the extent of absorption that are derived from serum concentration-time curves must be based on sampling periods of appropriate length or else be corroborated by urinary excretion data.

The serum concentration-time curves produced in the fedstate experiment are different from those produced in the fasting 
experiment because of the known retardation of digoxin absorption due to feeding before administration of drug (White et al., 1971). These results are similar to, and serve as a model for, those found when different oral formulations of digoxin were tested (Doherty, 1968; Lindenbaum et al., 1971; Manninen et al., 1971; Binnion and McDermott, 1972; Hibble et al., 1972). Our data could be falsely interpreted to indicate differences in bioavailability with respect to both the extent and rate of drug absorption. Such a conclusion is clearly contraindicated by the virtually identical urinary data in both experiments, suggesting identical extent of bioavailability in both settings.

Nonetheless, statements regarding digoxin bioavailability are still being made on the basis of peak serum levels alone (Butler, 1972; Lancet, 1972). The physician who must interpret data pertaining to the bioavailability of any drug in oral formulation, and of digoxin in particular, should be aware of the potential erroneous conclusions when only a single method of estimating bioavailability is used. The best studies would use at least three methods in the same subject and relate bioavailability to expected pharmacological effect.

We are grateful to Michele Sanda for performing the radioimmunoassays, to Dr. Thomas W. Smith for kindly supplying the antibody, and to members of the Division of Clinical Pharmacology, University of California Medical Center, for kindly volunteering as subjects.
Requests for reprints should be sent to: Dr. Kenneth L. Melmon, M-1089, Division of Clinical Pharmacology, University of California Medical Center, San Francisco, California 94143.

\section{References}

Binnion, P. F., and McDermott, M. (1972). Lancet, 2, 592.

Butler, V. P., jun. (1972). Progress in Cardiovascular Disease, 14, 571

Deutscher, R. N., Harrison, D. C., and Goldman, R. H. (1972). American fournal of Cardiology, 29, 47.

Dittert, L. W., Cressman, W. A., Kaplan, S. A., Riegelman, S., and Wagner, J. G. (1972). In Guidelines for Biopharmaceutical Studies in Man, p. 1. Washington D.C., American Pharmaceutical Association.

Doherty, J. E. (1968). American Fournal of Medical Science, 255, 382.

Doherty, J. E., Flanigan, W. J., and Dalrymple, G. V. (1972). American fournal of Cardiology, 29, 470.

Doherty, J. E., Hall, W. H., Murphy, M. L., and Beard, D. W. (1971). Chest, 59, 433 .

Hibble, A. G., Isaac, P., and Grahame-Smith, D. G. (1972). Lancet, 2, 90. Huffman, D. H., and Azarnoff, D. L. (1972). Fournal of the American Medical Association, 222, 957 .

Lancet, 1972, 2, 311 .

Lindenbaum, J., Mellow, M. H., Blackstone, M. O., and Butler, J. R., jun. (1971). New England fournal of Medicine, 285, 1344.

Manninen, V., Melin, J., and Gottfried, H. (1971). Lancet, 2, 934.

Oser, B. L. (1965). Hawk's Physiological Chemistry, p. 1233. New York, McGraw-Hill.

Smith, T. W., Butler, V. P., jun., and Haber, E. (1969). New England fournal of Medicine, 281, 1212 .

Wagner, J. G., et al. (1973). Fournal of the American Medical Association, 224, 199.

White, R. J., Chamberlain, D. A., Howard, M., and Smith, T. W. (1971). British Medical fournal, 1, 380.

\title{
Comparative Trial of a New Anticholinergic Bronchodilator, Sch I000, and Salbutamol in Chronic Bronchitis
}

\author{
H. POPPIUS, Y. SALORINNE
}

British Medical fournal, 1973, 4, 134-136

\section{Summary}

In 20 patients with chronic bronchitis and moderate or severe airways obstruction inhalation of $40 \mu \mathrm{g}$ of a new anticholinergic drug, Sch 1000, was found to produce stronger and more persistent bronchodilation than inhalation of $200 \mu \mathrm{g}$ of salbutamol.

\section{Introduction}

Sympathomimetics are thought to be more effective than anticholinergics in relieving bronchospasm in most asthmatics, and they are widely used in the treatment of patients with various kinds of obstructive lung diseases. In patients with chronic bronchitis, however, the bronchodilator effect of anticholinergic drugs is comparable to that of sympathomimetics (Altounyan, 1964; Crompton, 1968; Ulmer, 1971). Poppius et al. (1972) found that inhalation of a new anticholinergic drug, Sch 1000 (Boehringer Ingelheim), was followed by a bronchodilation response which was submaximal in a group of asthma patients but maximal in one of bronchitic patients-

\footnotetext{
Department of Pulmonary Diseases, University Central Hospital, 00290 Helsinki 29, Finland

H. POPPIUS, M.D., M.A.C.C.P., Senior Lecturer

Y. SALORINNE, M.D., Registrar
}

that is, subsequent inhalation of isoprenaline produced no further fall in airways resistance.

In the present study Sch 1000 was compared with salbutamol with regard to the bronchodilating effect in a group of patients with chronic bronchitis and moderate or severe airways obstruction.

\section{Material and Methods}

Twenty patients with chronic bronchitis (18 men and two women) were studied. The mean age was 55 (range 47-67) years. Care was taken to exclude patients with a history of asthma. In reply to the Medical Research Council's questionnaire on respiratory symptoms all patients were found to have persistent sputum (Q.15-17), 18 had periods of cough and sputum (Q.18), all had dyspnoea of at least grade 3 and 11 had dyspnoea of grade 5 (Q.19-24), and 17 had bouts of acute chest illness (Q.25). Only one patient gave a personal history and two a family history of atopy. Blood eosinophilia exceeding $5 \%$ was seen in one patient. Fifteen patients were cigarette smokers and four were ex-smokers. The forced expiratory volume in one second $\left(\mathrm{FEV}_{1}\right)$ and its proportion as a percentage of the vital capacity ( $\mathrm{FEV} \%$ ) are generally used as indices of ventilatory capacity. In this group the mean $\mathrm{FEV}_{1}$ was 1.33 (S.D. 0.41) 1 B.T.P.S. (body temperature and pressure, saturated with water vapour) for the men and $0.88,1$ B.T.P.S. for the women, corresponding to $42 \%$ (S.D. $14 \%$ ) and $36 \%$, (S.D. $8 \%$ ), respectively, of the predicted values (Poppius, 1965). All patients had an $\mathrm{FEV} \%$ value below $60 \%$, the mean $\mathrm{FEV} \%$ being $43 \%$. The mean increase in $\mathrm{FEV}_{1}$ after isoprenaline inhalation was $15 \%$ (S.D. $11 \%$ ). 